\title{
Atypical presentation of optic neuritis with unilateral inferior altitudinal visual field defect in multiple sclerosis
}

\author{
Cheau Wei Chin $\odot$, Mohammad Fathi Ismail \\ Department of Ophthalmology, Hospital Sultanah Aminah, Johor Bahru, Malaysia
}

\begin{abstract}
BACKGROUND: The purpose of this paper was to report a case of retrobulbar optic neuritis as the first manifestation of multiple sclerosis, with atypical presentations which include a sudden painless reduction in vision, without optic disc swelling and presence of unilateral inferior altitudinal visual field defect.

CASE REPORT: A 17-year-old girl presented to our clinic with right eye sudden painless drop in visual acuity to counting finger associated with headache. Her right eye optic nerve function tests were positive, but her optic disc was not swollen. Bjerrum's visual field chart noted right eye unilateral inferior altitudinal visual field defect with foveal involvement. Her blood investigations were normal, and magnetic resonance imaging (MRI) of brain and orbit was suggestive of multiple sclerosis. She was commenced on three days of high dose intravenous steroi, and was referred to neuromedical team for further management. At 3-month follow up, her right eye visual acuity improved to 6/6 with the restoration of normal colour vision.

CONCLUSIONS: In presence of atypical presentation of optic neuritis, multiple sclerosis must be thought of especially in young patients. Hence early co-management with neuromedical discipline is important to reduce the frequency and severity of attacks in the future.
\end{abstract}

KEY WORDS: retrobulbar optic neuritis; inferior altitudinal defect; multiple sclerosis; visual field

Ophthalmol J 2020; Vol. 5, 130-135

\section{INTRODUCTION}

Optic neuritis $(\mathrm{ON})$ refers to an inflammatory demyelinating disorder of the optic nerve, characterized by monocular, painful and subacute vision drop over a period of one to two weeks [1-3], commonly affecting young healthy females at mean age of 36 [4]. Rarely, ON may have atypical presentations such as the onset of fewes than 20 years old, painless and sudden onset of reduction in visual acuity $[1,2]$.

In addition, $\mathrm{ON}$ can present with any type of nerve-fibre-bundle-related visual field (VF) defect.
Keltner et al summarized that diffuse and central VF defect predominated in the affected eye at the first visit, then mainly partial arcuate, paracentral, and arcuate VF defects during follow-up [5]. Although the pattern of VF defect is not diagnostic of ON, altitudinal VF defect remains uncommon and should be further investigated for other aetiologies [2].

Demyelinating $\mathrm{ON}$ can occur in isolation or be associated with multiple sclerosis (MS) or neuromyelitis optica (NMO) [3]. Multiple sclerosis is an inflammatory demyelinating disease of the central nervous system characterized by multicentric in- 
flammation and myelin destruction. On the other hand, NMO is an antibody-mediated disorder of the central nervous system usually attacking the optic nerves and spinal cord, involving both gray and white matters [1].

In this article, we will discuss on the atypical presentations of $\mathrm{ON}$ and the uncommon visual field defects that may be present in $\mathrm{ON}$. We also suggest that MS should be suspected in the case of young healthy female and refer to neuromedical team early for co-management to further reduce the risk of recurrence.

\section{CASE REPORT}

A 17-year-old Malay girl who has no known medical illness, noticed a sudden painless drop in visual acuity of her right eye right after she woke up from her nap. It was accompanied by on and off dull frontal headache. She denies of ocular pain upon eye movement. Furthermore, she volunteered the history of recent viral flu one week ago, which lasted for four days, and has resolved at the time of presentation. There were no preceding flash or floaters, and patient denies of any trauma or insect bites. She also denies of body weakness, raised intracranial pressure symptoms, fever or any constitutional symptoms. She has no past ocular history and her family history was unremarkable.

On examination, her visual acuity was hand movement in the right eye and that was $6 / 6$ in the left eye. The relative afferent pupillary defect (RAPD) was grade I positive on the right eye, with a light brightness of $70 \%$ and red desaturation of $30 \%$. Ishihara colour vision test was unable to be performed over the right eye due to the poor vision. Her anterior segment and intraocular pressure were normal. Her optic discs were not swollen nor hyperemic and no retinitis patch nor vitritis seen in posterior pole. Her extraocular muscle movements were intact and painless, and no other cranial nerves affected. The rest of her neurological examination was unremarkable.

B-scan ultrasonography showed thickened right eye retroorbital optic nerve measuring $6.3 \mathrm{~mm}$ while that of the left eye measured $4.0 \mathrm{~mm}$. We proceeded with Bjerrum's screen chart as our patient had poor visual acuity and was unable to fixate on the stimulus target. Her Bjerrum's screen chart revealed right eye unilateral inferior altitudinal defect with foveal involvement, and the fellow eye showed supero-nasal arcuate defect (Fig. 1).

Her full blood count, renal function, erythrocyte sedimentation rate, C-reactive protein, folate and B12 level taken were within normal ranges. In addition, her serum anti-aquaporin-4 antibody returned negative. Her autoimmune screening and infective screening for venereal disease, human immunodeficiency virus (HIV), hepatitis B and C were non-reactive as well.

Magnetic resonance imaging (MRI) of brain and orbit reported multiple hyperintense lesions in pe-
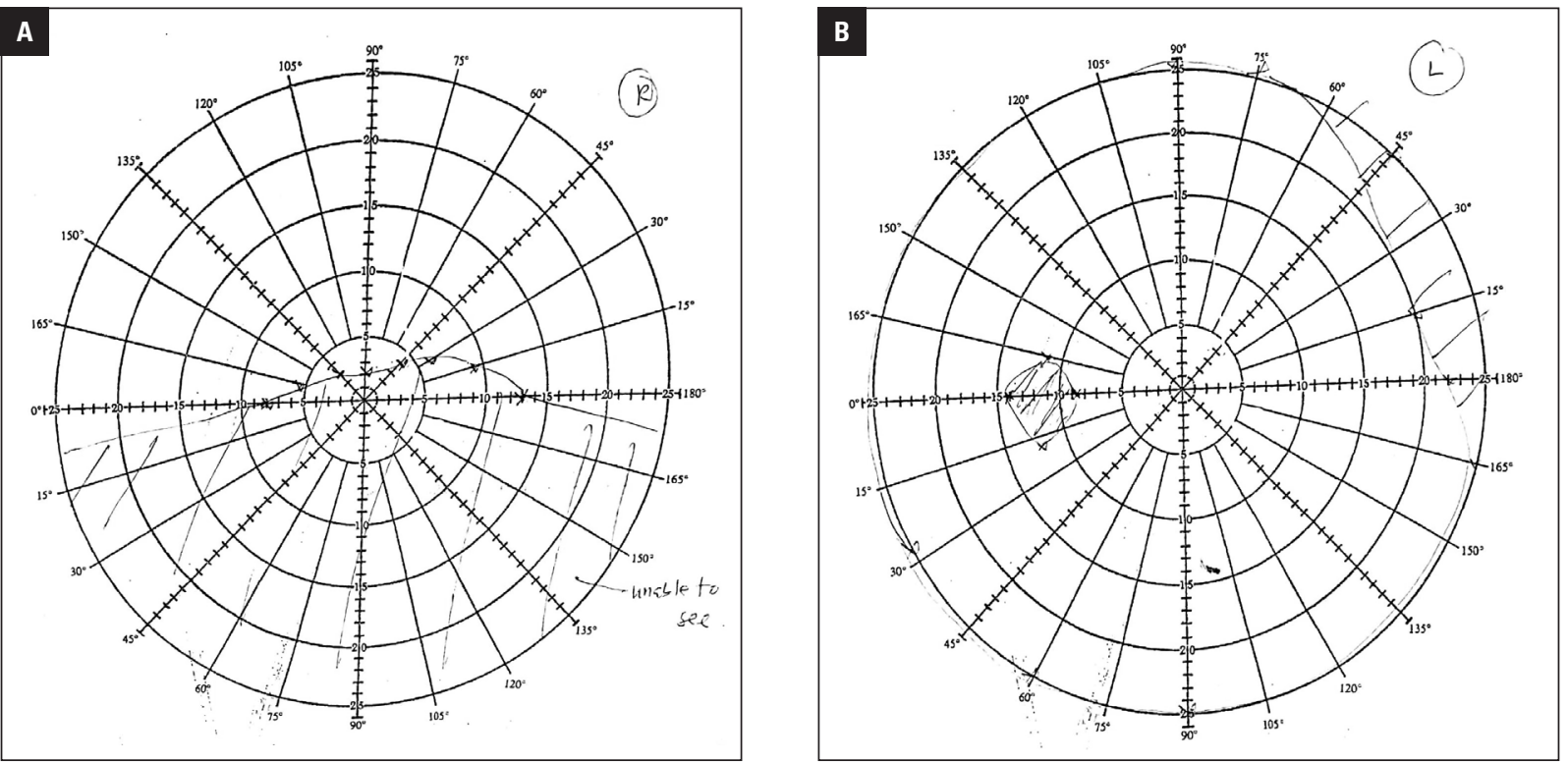

FIGURE 1. Bjerrum's Screen Chart of the (A) right and (B) left eye at initial visit 


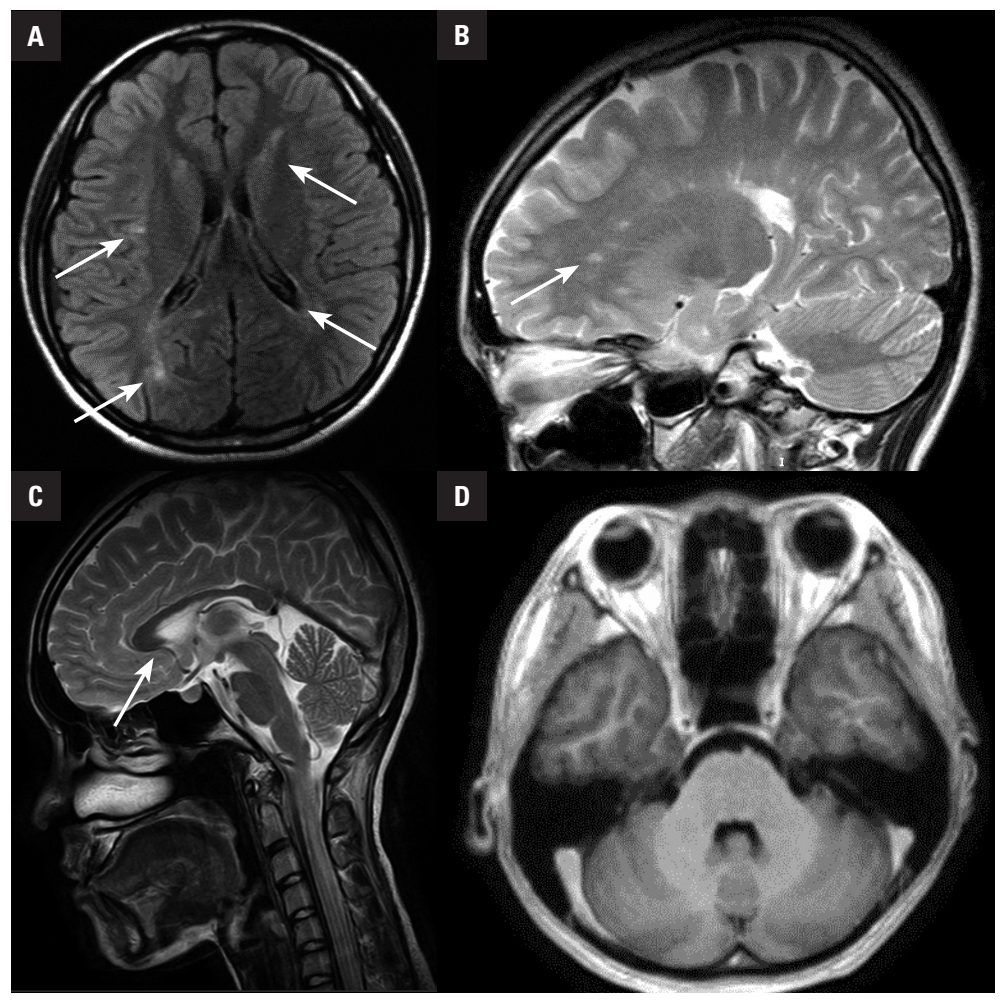

FIGURE 2. High intensity lesions at (A) periventricular, (B) juxtacortical and (C) pericallosal region

riventricular, juxtacortical and pericallosal region consistent with multiple sclerosis with dissemination in space, and no abnormal enhancement of optic nerve was seen (Fig. 2).

Furthermore, lumbar puncture was performed. The opening pressure was normal, and the cerebrospinal fluid (CSF) sample was clear, no signs of infection. Her CSF for anti-aquaporin-4 antibody was negative. On the other hand, her CSF electrophoresis revealed oligoclonal band with raised IgG index, which is consistent with multiple sclerosis.

Our patient was initially diagnosed with retrobulbar optic neuritis was secondary to viral flu in view of the history of recent flu prior to her presentation. However, the presence of bilateral visual field (VF) defect prompted us to proceed with contrast enhanced computerized tomography CECT brain and orbit to rule out the presence of space-occupying lesion. As there was no significant abnormality seen, we then proceeded with MRI brain and orbit, which suggested the diagnosis of multiple sclerosis. This is consistent with her CSF electrophoresis that revealed oligoclonal band with raised IgG index. This fulfils the Mcdonald criteria for the diagnosis of multiple sclerosis (MS) whereby she has one episode of attack, dissemination of space revealed by MRI and oligoclonal band present in CSF electrophoresis.

Other than MS, neuromyelitis optica spectrum disorder (NMOSD) is also commonly associated with $\mathrm{ON}$ in young healthy females. However, this was ruled out by the absence of anti-aquaporin- 4 antibody in both serum and CSF. Although painless monocular vision loss and unilateral inferior altitudinal defect also paints a picture of non-arteritic anterior ischemic optic neuropathy (NAION), this does not fit her age group and there is lack of ischemic risk factors such as diabetes, hypertension or any history of major surgery to justify the diagnosis.

She was referred to neuromedical team, and was started on methylprednisolone $250 \mathrm{mg}$ i.v. 6-hourly for 3 days, subsequently discharged home with oral Prednisolone $1 \mathrm{mg} / \mathrm{kg} /$ day and slowly tapered over a period of 6 weeks as per neuromedical plan. Her right eye visual acuity has improved to $3 / 60$ after completing 3 days of methylprednisolone treatment.

At 3-month follow up, her vision has improved to $6 / 6$ both eyes, with normal colour vision. There was no more relative afferent pupillary defect (RAPD) present. Her anterior segment, intraocular pressure and posterior segment were normal, no 

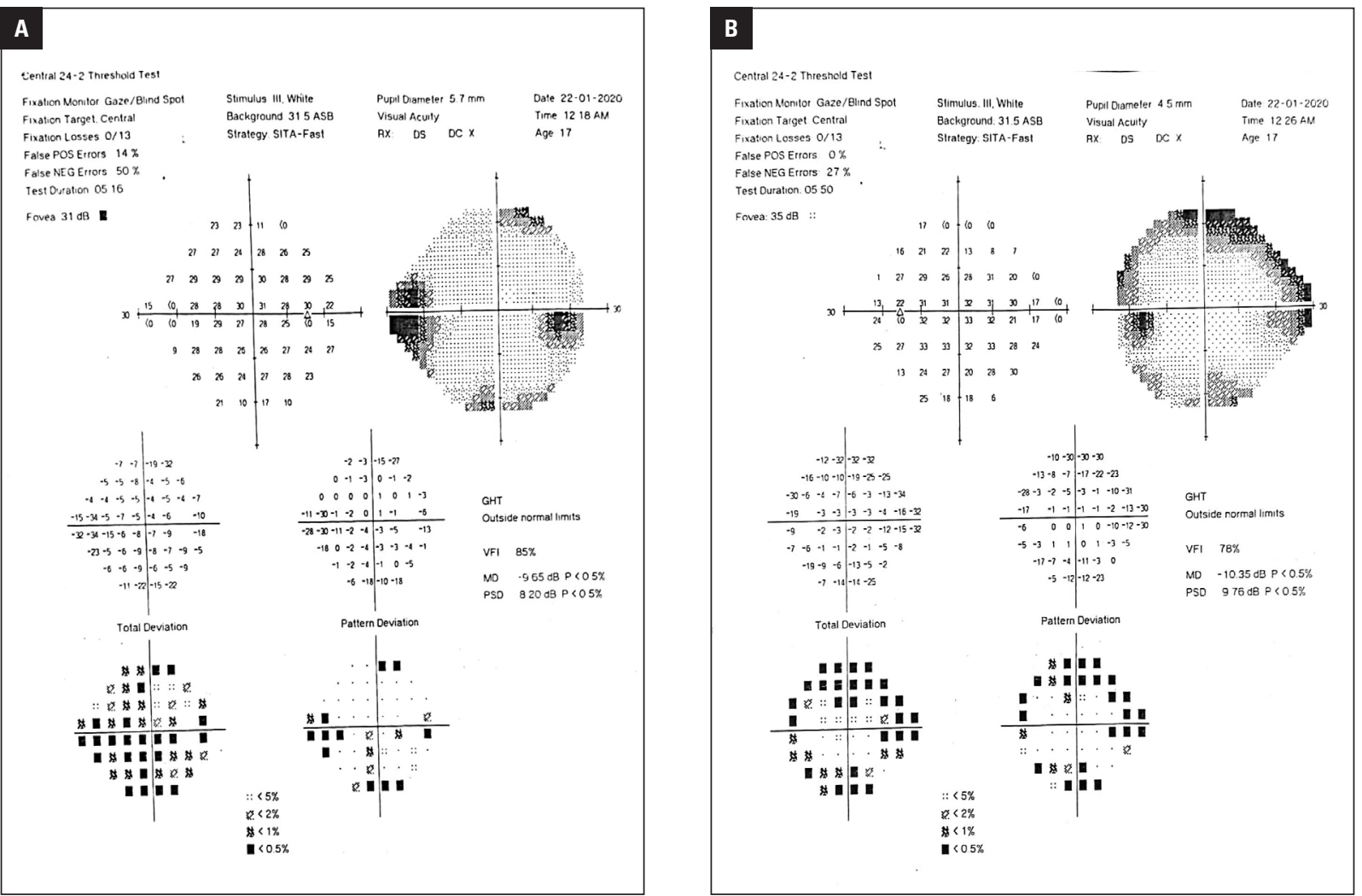

FIGURE 3. Humphrey visual field 24-2 of (A) right eye and (B) left eye at 3-month follow-up

disc pallor nor swelling noted. This time around we monitored her VF defect with Humphrey's visual field. Her right eye VF has cleared up leaving nasal step defect with some patchy loss, and left eye revealed superior arcuate defect (Fig. 3).

\section{DISCUSSION}

Wilhelm and Schabet [4] described a typical presentation of demyelinating $\mathrm{ON}$ as occurrence between age 18 to 50 , unilateral, peribulbar pain, and improvement of vision after treatment. The mean age of onset is 36 , and demyelinating $\mathrm{ON}$ is rare in patients below 18 years old. According to Kale [2], only $8 \%$ of patients with ON presented with painless vision loss, which is similar to our patient's presentation. This could be explained by the inflammation lying in the intracranial portion of optic nerve [4]. Gaier et al [1] and Hoorbakht et al [7] suggested that painless visual loss should raise suspicion for other causes such as non-arteritic anterior ischemic optic neuropathy (NAION) or Leber's hereditary optic neuropathy (LHON). However, both diagnoses did not fit our patient's age, gender, and risk factors.
Optic neuritis can be divided into:

- 1 - retrobulbar $\mathrm{ON}$ with normal disc appearance;

-2 - papillitis involving the anterior aspect of the optic nerve with optic disc swelling;

-3 - perineuritis with the involvement of the optic nerve sheath;

- 4 - neuroretinitis, with optic disc swelling and macular star [3]. Among these subtypes, retrobulbar and papillitis $\mathrm{ON}$ are more commonly associated with MS. ON that present in isolation accounts for $20 \%$ of the initial presentation of MS [2]. From the Optic Neuritis Treatment Trial (ONTT) [8], we learnt that the patients presented with various types of VF defect, predominantly diffuse $(66 \%)$ and central field loss $(27 \%)$ on the affected eye during their first presentation. Our patient presented with unilateral inferior altitudinal field defect on the affected eye during initial presentatio, and then transformed into a nasal step-defect during follow up. This is uncommon as Keltner et al [5] concluded that only $8 \%$ of the participants from the ONTT presented with altitudinal field defect initially, and no more than $2 \%$ developed nasal step defect during their follow up. 
Furthermore, Keltner et al [5] revealed that 75\% of the patients developed VF defect on the fellow eye during follow up, predominantly partial arcuate, paracentral, and arcuate defects. In this case, our patient developed superior arcuate defect over her fellow eye, suggesting the involvement of the optic nerve fibre bundle. Despite the limited use of VF in aiding diagnosis, Kale et al [2] suggested that the presence of altitudinal defect warrant consideration of other differential diagnosis. In this case, we have proceeded with urgent brain imaging and managed to rule out space occupying lesions and life-threatening intracranial pathologies.

In 1970, Hayreh [9] suggested that altitudinal VF defect without retinal lesion is caused by disruption in the posterior ciliary artery circulation. Despite the pathophysiology of $\mathrm{ON}$ remains unclear, it is postulated to result from inflammation and demyelination of the optic nerve [3]. Cytokines and other inflammatory mediators are released when activated peripheral T-cells cross the blood brain barrier, causing destruction of myelin, neural cell death and axonal degeneration [3, 7]. Here we suspect that either the inflammation of the optic nerve itself or the secondary perfusion defect caused by the inflammation of the optic nerve has resulted in an altitudinal VF defect in our patient.

Brain MRI is helpful diagnostically and prognostically to determine the risk of developing MS for patients with ON based on the number of demyelinating white matter lesions [3]. ONTT concluded that the risk of patients with ON developing MS by 15 years was $25 \%$ if their MRI brain was normal at time of diagnosis, and the risk triples to $78 \%$ if their MRI brain has more than three lesions [8]. In our patient's circumstance, she has lesions at periventricular, juxtacortical and pericallosal regions, and CSF electrophoresis has confirmed her diagnosis of MS.

However, no enhancement of optic nerve was seen in our patient's MRI. According to Kupersmith et al [10], enhancement of the optic nerve has a sensitivity of $94 \%$ in acute optic neuritis, and only $5 \%$ of patients with ON did not have enhancement of the affected optic nerve. Nonetheless, there wae no significant difference in terms of visual acuity and colour vision between those with and without optic nerve enhancement [10].

Intravenous methylprednisolone (IVMP) is recommended when there is monocular involvement, significant bilateral visual loss, or when fast recovery to normal visual acuity is required [3,
7]. Once systemic infection is ruled out, IVMP in a dose of $250 \mathrm{mg}$ 6-hourly should be commenced over a 3-day course, followed by 11 days of daily oral prednisone $(1 \mathrm{mg} / \mathrm{kg} /$ day $)$ as per recommended by ONTT [8]. The main aim of treatment is to reduce the frequency and severity of attacks, and prevention of axonal loss in both $\mathrm{ON}$ and MS [7].

At 1-year follow-up, 94\% of the patients who received IVMP in ONTT regained vision of $6 / 12$ or better but subtle symptoms such as blurred vision may persist even with VA of $>6 / 6$ [7]. Our patient has already achieved visual acuity of $6 / 6$ with normal colour vision at 3-month follow-up.

\section{CONCLUSION}

Optic neuritis is a disease with many faces. Atypical presentations such as painless acute vision loss prompt other differential diagnoses but this does not rule out ON. It may also present with inferior altitudinal VF defect, which warrants further imaging to exclude other intracranial pathologies as this is an uncommon presentation. Brain MRI is important diagnostically and prognostically in monitoring disease progression. Last but not least, multiple sclerosis should be suspected in the case of young healthy female as early diagnosis and early co-management with neuromedical team reduces the frequency and severity of recurrence.

\section{Consent for publication}

The case patient had consented for publication of this case report, including the usage of her fundus photograph, OCT scans and recorded video of the surgery.

\section{Conflicg of interests}

The authors declare that there is no conflict of interest.

\section{Funding acknowledgement}

The authors received no financial support for the research, authorship, and/or publication of this article.

\section{REFERENCES}

1. Gaier ED, Boudreault K, Rizzo JF, et al. Atypical Optic Neuritis. Curr Neurol Neurosci Rep. 2015; 15(12): 76, doi: 10.1007/s11910-0150598-1, indexed in Pubmed: 26467052.

2. Kale N. Optic neuritis as an early sign of multiple sclerosis. Eye Brain. 2016; 8: 195-202, doi: 10.2147/EB.S54131, indexed in Pubmed: 28539814. 
3. Pau D, Al Zubidi Nu, Yalamanchili S, et al. Optic neuritis. Eye. 2011; 25(7): 833-842, doi: 10.1038/eye.2011.81, indexed in Pubmed: 21527960.

4. Wilhelm H, Schabet M. The Diagnosis and Treatment of Optic Neuritis. Dtsch Arztebl Int. 2015; 112(37): 616-25; quiz 626, doi: 10.3238/ arztebl.2015.0616, indexed in Pubmed: 26396053.

5. Keltner JL, Johnson CA, Cello KE, et al. Optic Neuritis Study Group. Visual field profile of optic neuritis: a final follow-up report from the optic neuritis treatment trial from baseline through 15 years. Arch Ophthalmol. 2010; 128(3): 330-337, doi: 10.1001/archophthalmol.2010.16, indexed in Pubmed: 20212204.

6. Trapp BD, Peterson J, Ransohoff RM, et al. Axonal transection in the lesions of multiple sclerosis. N Engl J Med. 1998; 338(5): 278-285, doi: 10.1056/NEJM199801293380502, indexed in Pubmed: 9445407.
7. Hoorbakht $H$, Bagherkashi F. Optic neuritis, its differential diagnosis and management. Open Ophthalmol J. 2012; 6: 65-72, doi: 10.2174/ 1874364101206010065, indexed in Pubmed: 22888383.

8. Optic Neuritis Study Group. Multiple sclerosis risk after optic neuritis: final optic neuritis treatment trial follow-up. Arch Neurol. 2008; 65(6): 727-732, doi: 10.1001/archneur.65.6.727, indexed in Pubmed: 18541792.

9. Hayreh SS. Pathogenesis of visual field defects. Role of the ciliary circulation. Br J Ophthalmol. 1970; 54(5): 289-311, doi: 10.1136/ bjo.54.5.289, indexed in Pubmed: 4987892.

10. Kupersmith MJ, Alban T, Zeiffer B, et al. Contrast-enhanced MRI in acute optic neuritis: relationship to visual performance. Brain. 2002; 125(Pt 4): 812-822, doi: 10.1093/brain/awf087, indexed in Pubmed: 11912114. 